\title{
Comorbidities Associated with Worse Outcomes Among Inpatients Admitted for Acute Gastrointestinal Bleeding
}

\author{
K. Siebenhüner ${ }^{1,2,3,7}$ (D) J. Blaser ${ }^{4} \cdot$ A. Nowak $^{5,6}$ (D) M. Cheetham ${ }^{7} \cdot$ B. U. Mueller ${ }^{7} \cdot$ E. Battegay $^{2,3,7} \cdot$ P. E. Beeler ${ }^{1,7}$ (D)
}

Received: 27 September 2020 / Accepted: 25 July 2021 / Published online: 7 August 2021

(c) The Author(s) 2021

\begin{abstract}
Background Multimorbidity increases healthcare resource utilization. Little is known on specific comorbidity combinations. Aims To identify comorbidities associated with increased resource utilization among inpatients admitted for gastrointestinal bleeding (GIB).

Methods This retrospective cross-sectional study, 1/2010-5/2018 at the University Hospital Zurich, Switzerland, analyzed electronic health records of patients with upper (UGIB) and lower (LGIB) GIB, focusing on length of stay (LOS) and 30-day readmissions for resource use and clinical outcomes, investigated by multivariable regression adjusted for antithrombotics. Results Of 1101 patients, 791 had UGIB and 310 LGIB, most often melena and bleeding diverticula, respectively. In UGIB, thromboembolic events showed a trend toward $27 \%$ increased LOS $(1.27 ; 95 \%$ confidence interval [CI] 1.00-1.61), antithrombotics independently associated with $46 \%$ increased LOS (1.46; 95\% CI 1.32-1.62). Cancer (odds ratio [OR] 2.86; 95\% CI 1.68-4.88) independently associated with 30-day readmissions, anemia showed a trend (OR 1.68; 95\% CI 1.00-2.84). In LGIB, none of the investigated comorbidities associated with increased LOS, but antithrombotics independently associated with $25 \%$ increased LOS (1.25; 95\% CI 1.07-1.46). Atrial fibrillation/flutter (OR 2.69; 95\% CI 1.06-6.82) and cancer (OR 4.76; 95\% CI 1.40-16.20) associated strongly with 30-day readmissions.

Conclusions In both groups, cancer associated with 30-day readmissions, antithrombotics with increased LOS. Thromboembolic events and anemia showed clinically important trends in UGIB. Atrial fibrillation/flutter associated with 30-day readmissions in LGIB. Prospective studies are needed to investigate these complex multimorbid populations and establish appropriate guidelines.
\end{abstract}

Keywords Comorbidity $\cdot$ Gastrointestinal bleeding $\cdot$ Inpatients $\cdot$ Length of stay $\cdot$ Multimorbidity $\cdot$ Patient readmission

K. Siebenhüner

klarissa.siebenhuener@uzh.ch

1 Institute of Epidemiology, Biostatistics and Prevention, University of Zurich, Hirschengraben 84, 8001 Zurich, Switzerland

2 Department of Internal Medicine, University Hospital of Zurich, Zurich, Switzerland

3 Centre of Competence Multimorbidity, University of Zurich, Zurich, Switzerland

4 Directorate of Research and Education, University Hospital of Zurich, Zurich, Switzerland

5 Department of Endocrinology and Clinical Nutrition, University Hospital Zurich and University of Zurich, Zurich, Switzerland

6 Department of Internal Medicine, Psychiatry University Hospital Zurich, Zurich, Switzerland

7 Department of Internal Medicine, University Hospital Zurich and University of Zurich, Zurich, Switzerland

\author{
Abbreviations \\ EHR Electronic health record \\ GIB Gastrointestinal bleeding \\ ICD International classification of diseases \\ LOS Length of stay \\ LGIB Lower gastrointestinal bleeding \\ UGIB Upper gastrointestinal bleeding
}

\section{Introduction}

The aging population of patients with multiple concomitant acute and chronic conditions is growing [1]. An increasing proportion of patients is considered multimorbid due to the effects of progressive deterioration in conditions often relating to the heart, kidney and liver [2]. Van den Akker and colleagues [3] specified multimorbidity as the simultaneous presence of two or more chronic diseases. However, the need 
for a standardized definition is advocated [4]. More recent approaches emphasize the characterization of multimorbidity in terms of the complexity of disease combinations [5]. For instance, people over 64 years of age with dementia have a significantly higher number of comorbidities (most often diabetes or other cardiovascular diseases like hypertension) than people who are not diagnosed with this index disease [6].

The WHO estimates the average life expectancy of Europeans at 77.5 years [7]. With improving control of chronic diseases, both life expectancy and numbers of multimorbid patients will increase further [8]. Multimorbidity is also the rule rather than the exception in Switzerland, both in hospital and especially in general medicine, and it increases with age [9].

Medical and socio-economic healthcare needs are expected to be a major task within the next decades [10]. However, disease outbreaks such as the coronavirus disease 2019 (COVID-19), which is especially threatening to multimorbid patients, add new challenges to the existing burdens on the healthcare system [11]. A part of the risk assessment for the management of multimorbid patients is the consideration of risk factors associated with worse clinical outcomes in order to improve further resource planning and monitoring. Hospital length of stay (LOS) and 30-day readmissions represent important clinical outcomes with implications for healthcare utilization and costs. For instance, a recent study has shown that ancillary depression in multimorbid inpatients is associated with increased LOS and 30-day readmissions [12].

The present study investigated inpatients at a Swiss tertiary care medical center who were hospitalized for the treatment of acute gastrointestinal bleeding (GIB). GIB requires frequent hospitalizations with esophageal varices, peptic ulcer disease, esophagitis and gastritis as most common causes for upper gastrointestinal bleeding (UGIB) [13] and diverticular bleeding for lower gastrointestinal bleeding (LGIB) [14].

GIB is associated with a burden of disease, with UGIB having a reported mortality of $2-10 \%$ [15], and LGIBalthough less frequent-a mortality of $10-20 \%$ [16]. The objective of this study was to determine whether UGIB or LGIB was associated with the two important clinical outcomes, increased LOS and 30-day readmissions depending on clinically significant comorbidities.

Thus, the purpose of our study was to:

(a) describe and characterize a large inpatient population admitted for the treatment of acute GIB, stratified by UGIB vs LGIB, and

(b) to investigate comorbid conditions in UGIB vs LGIB populations that are associated with increased LOS and 30-day readmissions.

\section{Methods}

\section{Design and Study Period}

We conducted a retrospective, cross-sectional study using electronic health record (EHR) data and considered eligible inpatients hospitalized at the University Hospital Zurich between January 1, 2010, and May 31, 2018 (total of 273,378 inpatient stays). All EHR data used in this study were routinely collected. We followed the STrengthening the Reporting of OBservational studies in Epidemiology (STROBE) guidelines [17].

\section{Ethical Considerations}

Ethics approval was obtained from the Cantonal Ethics Commission Zurich, BASEC 2016-00525, which waived the requirement for obtaining informed consent for this retrospective study.

\section{Setting}

The University Hospital Zurich is a large tertiary care academic medical center in Zurich, Switzerland. The institution has approximately 900 beds and cares for more than 35,000 inpatients per year. Over 8200 employees work at this hospital, covering all specialties except orthopedic surgery and pediatrics.

\section{Inclusion and Exclusion Criteria}

The reason for hospitalization was the primary diagnosis, flagged as such in the EHR. All diagnoses were ICD-10 coded (ICD: International Classification of Diseases, WHO, Geneva, Switzerland). The ICD-10 coded primary diagnoses were used to identify our population of interest: patients admitted for the treatment of acute GIB. Iterative literature searches were alternated with research group discussions to reach consensus about the ICD-10 codes that referred to these patients. The same process was used to define the ICD-10 codes for the identification of clinically relevant comorbidities among secondary diagnoses. After the study population was identified, detailed chart reviews were conducted to clarify any case of ambiguity: For instance, the diagnoses sometimes indicated that a patient suffered from both UGIB and LGIB. Therefore, the ICD-10 code K92.2 (gastrointestinal hemorrhage) was verified by chart review as UGIB; except for 7 cases, which were excluded due to concurrent diagnoses indicating LGIB (i.e., hemorrhoids, diverticulitis and diverticulosis with bleeding). The ICD10 code K92.1 (melena) was allocated to UGIB [18]. If 
potential subjects had a record that indicated refusal to be involved in research, they were excluded from the study. Patients with incomplete information on potential readmission events (patients discharged within 29 days before end of study period) and patients who died during their stay were also excluded $(n=59)$.

\section{Definition and Classification of Gastrointestinal Bleeding}

We used ICD-10 coded diagnoses to identify patients with acute GIB and stratified them into two study groups.

I. UGIB: I85.0, I98.3, K22.3, K22.6, K22.8, K25.0, K25.2, K25.4, K25.6, K26.0, K26.2, K26.4, K26.6, K27.0, K27.2, K27.4, K27.6, K28.0, K28.4, K28.6, K29.0, K31.82, K92.0, K92.1 and K92.2 and

II. LGIB: K55.22, K55.82, K57.01, K57.03, K57.11, K57.13, K57.31, K57.41, K57.51, K57.91, K57.93, K62.5, K64.0, K64.1, K64.2, K64.3 and K64.9

\section{Outcomes and Measures}

The outcome variables investigated were LOS and 30-day all-cause readmissions, the latter defined as any admission within 30 days of the index admission. The predefined exposure of interest variable was presence of 15 comorbid conditions potentially associated with increased LOS or 30-day readmissions (cf. section Co-Variables, below). We strictly differentiated between UGIB and LGIB.

We used the natural logarithm to transform the skewed outcome variable LOS, as we did previously (described in more detail elsewhere [12]). This allowed the application of linear regression models. The estimated coefficients were back-transformed by raising e to the power of the coefficients, and these results can be interpreted as percentage increases or decreases.

\section{Co-variables}

Co-variables were considered according to a list of diseases with clinical relevance, using the bleeding risk score termed HAS-BLED Score [19] and the stroke risk categorization schema CHA2DS2-VASCc-Score [20]. The 15 comorbidities were identified among all secondary diagnoses which were comprehensively ICD-10 coded. The physicians in charge of the patients defined and updated the primary diagnosis and all secondary diagnoses over the course of the hospital stay. After the patients discharge, professional medical coders assigned ICD-10 codes to each diagnosis. Four conditions were based on the ICD-10 coded Elixhauser Comorbidity Index definitions by Quan et al. [21]: anemia (blood loss and iron deficiency), cancer (solid tumor with/without metastasis), coagulopathy (purpura and other hemorrhagic diatheses) and chronic pulmonary diseases. The other 11 conditions (i.e., alcohol use disorders, atrial fibrillation or flutter, chronic kidney disease, depression, diabetes mellitus, heart failure, heart valve disease, hypertension, liver disease, thromboembolic event and vascular disease) were defined by means of iterative literature searches alternated with research group discussions to reach consensus. Thromboembolic event includes the diagnoses pulmonary embolism and deep vein thrombosis. Vascular disease was defined by the diagnoses of acute myocardial infarction, coronary heart disease, chronic ischemic heart disease and peripheral arterial vascular disease. The comprehensive lists with their specific ICD-10 codes are summarized in Supplementary Table S1. Multimorbidity was defined as the presence of at least two diagnoses [22]. We controlled for multimorbidity and burden of disease by adjusting for diagnosis count [23].

To control for potential confounding in the context of the therapeutic conflict of reinstating antithrombotic medication for our inpatients admitted with acute GIB, comprehensive drug ordering data were extracted. Antithrombotic agents were identified by the Anatomical Therapeutic Chemical Classification System (ATC, World Health Organization, Geneva, Switzerland) class B01A of which the following categories of drugs were ordered at least once for the inpatients studied: vitamin K antagonists (ATC class B01AA), heparin (ATC class B01AB), platelet aggregation inhibitors excluding heparin (ATC class B01AC) and direct factor Xa inhibitors (ATC class B01AF). All drug prescriptions including "free-text" orders were part of the data analysis: We (i) manually mapped the most frequent orders lacking ATC information to the correct ATC code, (ii) used an automated character sequence comparison algorithm to map the same ATC code to similar orders and (iii) reviewed the automated mappings and revised where necessary. These steps were repeated several times to augment and improve our mapping table.

\section{Data Processing and Statistics}

Descriptive statistics of all measures were generated with median and interquartile ranges for continuous variables with a non-parametric distribution. For categorical variables, numbers and percentages of the total are reported. We conducted comparative statistical analyses to describe patients with UGIB vs LGIB using chi-squared tests for categorical variables and Mann-Whitney U tests for continuous variables. For all patients with UGIB and LGIB, we used multivariable linear and logistic regression models to test whether the 15 conditions were independently associated with longer LOS and 30-day readmissions, respectively. All models were adjusted for age, sex, diagnosis count, year of 
discharge, the 15 comorbidities, and orders of antithrombotic medications. We additionally controlled for LOS in our 30-day readmission models. For the statistical analyses, the software R, version 3.6.1 (R Foundation for Statistical Computing, Vienna, Austria) was used. A $p$ value of $\leq 0.05$ was considered as significant.

\section{Results}

A total of 1101 patients admitted for the treatment of acute GIB were identified, and two-thirds of them were male (Table 1). The proportions of patients with UGIB and LGIB were $72 \%$ and $28 \%$, respectively. The UGIB population was significantly younger (median 65 years) than the patients with LGIB (median 71 years). All included patients were multimorbid. Regarding comorbidities, the proportions of patients with vascular disease, alcohol use disorder, atrial fibrillation/flutter, cancer and liver disease differed significantly between the study groups. A total of 671 patients $(61 \%)$ received antithrombotic medications for the prevention of thromboembolic and cardiovascular events. Over the course of their stays, $10 \%$ of all patients admitted for the treatment of acute GIB received vitamin $\mathrm{K}$ antagonists, $44 \%$ heparin, $31 \%$ had platelet aggregation inhibitors, and $<3 \%$ received direct factor Xa inhibitors. The median length of stay was 6 days for UGIB compared with 5 days for LGIB. Overall, $17 \%$ of all patients with GIB were readmitted to our institution within 30 days after discharge.

UGIB patients were most often admitted for melena, whereas bleeding diverticula were the predominant reason for admission among LGIB patients (Table 2).

\section{Analysis of Associations with LOS Among UGIB Patients}

LOS appeared to increase by $10 \%$ per additional diagnosis in UGIB patients (Table 3). Thromboembolic events showed a clinically important trend toward increased LOS which almost reached statistical significance. Antithrombotic agents were independently associated with $46 \%$ longer LOS.

\section{Analysis of Associations with 30-Day Readmissions Among UGIB Patients}

Patients were more likely of having a 30-day readmission with increasing year during the study period (Table 3). Cancer was the only comorbidity statistically significantly associated with a 30-day readmission, with an odds ratio almost three times as high as for UGIB patients without cancer. Clinically important trends toward 30-day readmissions were observed for patients with hypertension, diabetes, chronic kidney disease, chronic obstructive pulmonary disease (COPD) and finally anemia the only one nearly reaching statistical significance.

\section{Analysis of Associations with LOS Among LGIB Patients}

None of the investigated comorbidities were associated with an increased LOS. However, older patients stayed longer in the hospital and patients seemed to stay almost $10 \%$ longer per additional diagnosis. Heart valve disease and vascular disease were statistically significantly associated with a shorter LOS (Table 4). Antithrombotic agents were independently associated with a $25 \%$ increased LOS.

\section{Analysis of Associations with 30-Day Readmissions Among LGIB Patients}

Atrial fibrillation/flutter and cancer were independently associated with 30-day readmissions with nearly three and nearly five times increased odds ratios, respectively. Potentially important trends toward 30-day readmissions were observed for patients with anemia, alcohol use disorders, vascular disease and also for patients on anticoagulants; however, none of those close to statistical significance (Table 4).

\section{Discussion}

In this retrospective study of 1101 patients admitted for the treatment of GIB, we found higher presentation of UGIB compared to LGIB among patients with a multimorbidity profile. While thromboembolic events only showed a clinically important trend toward increased LOS in patients with UGIB, antithrombotic agents statistically significantly associated with increased LOS. Among patients with LGIB, none of the comorbidities investigated were associated with increased LOS; however, atrial fibrillation/flutter was strongly associated with 30-day readmissions. Cancer showed strong independent associations with 30-day readmissions in both, UGIB and LGIB. The present investigation revealed a relatively high 30 -day readmission rate of $18.1 \%$ among patients with UGIB versus $14.5 \%$ among LGIB patients. We further found multimorbidity, i.e., the burden of disease considered by diagnosis count, to be an important independent reason for a prolongation of LOS. LOS seemed to increase by approximately $10 \%$ per additional diagnosis for both groups.

GIB is common [24, 25]. Clinical symptoms range from anemia to fulminant bleeding with shock. Management requires a differentiated approach from the first sign of GIB, during acute care and for the prevention of recurrent bleeding. Multimorbidity increases the complexity of patient care 
Table 1 Baseline characteristics

Upper GI bleeding $(n=791) \quad$ Lower GI bleeding $(n=310) \quad P$ value $\quad$ GI patients (overall, $n=1101)$

\begin{tabular}{|c|c|c|c|c|}
\hline \multicolumn{5}{|l|}{ Demographics } \\
\hline Age, median years [IQR] & $65.0[52.0,75.0]$ & $71.00[61.0,79.0]$ & $<0.001$ & $67.0[54.0,76.0]$ \\
\hline Male sex, $n(\%)$ & $519(65.6)$ & $199(64.2)$ & 0.708 & $718(65.2)$ \\
\hline \multicolumn{3}{|l|}{ Marital status } & \multicolumn{2}{|l|}{0.181} \\
\hline Married/partnered (4) & $418(52.8)$ & $165(53.2)$ & & $583(53.0)$ \\
\hline Widowed/divorced/separated (3) & $204(25.8)$ & $92(29.7)$ & & $296(26.9)$ \\
\hline Single (2) & $144(18.2)$ & $41(13.2)$ & & $185(16.8)$ \\
\hline Other/unknown (1) & $25(3.2)$ & $12(3.9)$ & & $37(3.4)$ \\
\hline Number of diagnoses, median [IQR] & $9.0[6.0,13.0]$ & $9.00[5.0,13.0]$ & 0.941 & $9.00[6.0,13.0]$ \\
\hline$\geq 2$ ICD-10 codes & $42(5.3)$ & $30(9.7)$ & & $72(6.5)$ \\
\hline $3-5$ ICD-10 codes & $140(17.7)$ & $52(16.8)$ & & $192(17.4)$ \\
\hline 6-9 ICD-10 codes & $255(32.2)$ & $81(26.1)$ & & $336(30.5)$ \\
\hline$\geq 10$ ICD-10 codes & $354(44.8)$ & $147(47.4)$ & & $501(45.5)$ \\
\hline \multicolumn{5}{|l|}{ Comorbidities $* n(\%)$} \\
\hline Hypertension ${ }^{\mathrm{a}, \mathrm{b}}$ & $239(30.2)$ & $108(34.8)$ & 0.158 & $347(31.5)$ \\
\hline Vascular disease $\mathrm{e}^{\mathrm{b}}$ & $199(25.2)$ & $111(35.8)$ & 0.001 & $310(28.2)$ \\
\hline Alcohol use disorders ${ }^{\mathrm{a}}$ & $248(31.4)$ & $25(8.1)$ & $<0.001$ & $273(24.8)$ \\
\hline Coagulopathy & $156(19.7)$ & $70(22.6)$ & 0.33 & $226(20.5)$ \\
\hline Chronic kidney disease $^{\mathrm{a}}$ & $152(19.2)$ & $71(22.9)$ & 0.199 & $223(20.3)$ \\
\hline Diabetes mellitus ${ }^{\mathrm{b}}$ & $152(19.2)$ & $71(22.9)$ & 0.199 & $223(20.3)$ \\
\hline Atrial fibrillation/flutter & $104(13.1)$ & $68(21.9)$ & $<0.001$ & $172(15.6)$ \\
\hline Anemia & $104(13.1)$ & $49(15.8)$ & 0.294 & $153(13.9)$ \\
\hline Cancer & 94 (11.9) & $19(6.1)$ & 0.007 & $113(10.3)$ \\
\hline Valvular heart disease ${ }^{\mathrm{b}}$ & $61(7.7)$ & $35(11.3)$ & 0.076 & $96(8.7)$ \\
\hline Liver disease $^{\mathrm{a}}$ & $79(10.0)$ & $12(3.9)$ & 0.001 & $91(8.3)$ \\
\hline Chronic pulmonary disease & $57(7.2)$ & $28(9.0)$ & 0.37 & $85(7.7)$ \\
\hline Heart failure/insufficiency ${ }^{\mathrm{b}}$ & $48(6.1)$ & $22(7.1)$ & 0.623 & $70(6.4)$ \\
\hline Depression & $34(4.3)$ & $11(3.5)$ & 0.692 & $45(4.1)$ \\
\hline Thromboembolic event $^{\mathrm{b}}$ & $29(3.7)$ & $6(1.9)$ & 0.2 & $35(3.2)$ \\
\hline Antithrombotic agents & $468(59.2)$ & $203(65.5)$ & 0.062 & $671(60.9)$ \\
\hline Vitamin K antagonists (ATC B01AA) & $75(9.5)$ & 39 (12.6) & 0.159 & $114(10.4)$ \\
\hline Heparin group (ATC B01AB) & $345(43.6)$ & $138(44.5)$ & 0.839 & $483(43.9)$ \\
\hline $\begin{array}{l}\text { Platelet aggregation inhibitors excl. Heparin } \\
\text { (ATC B01AC) }\end{array}$ & $231(29.2)$ & $110(35.5)$ & 0.051 & $341(31.0)$ \\
\hline Direct factor Xa inhibitors (ATC B01AF) & $16(2.0)$ & $12(3.9)$ & 0.124 & $28(2.5)$ \\
\hline \multicolumn{5}{|l|}{ Outcomes } \\
\hline Length of stay in days, median [IQR] & $6.0[4.0,10.0]$ & $5.00[4.0,9.0]$ & 0.378 & $6.0[4.0,10.0]$ \\
\hline 30-day readmission, $n(\%)$ & $143(18.1)$ & $45(14.5)$ & 0.186 & $188(17.1)$ \\
\hline
\end{tabular}

A list of 15 comorbidities was defined according to ICD-10 diagnosis codes. The following diagnoses were based on the Elixhauser Comorbidity Index [21]: anemia, cancer, coagulopathy and chronic pulmonary diseases. Patients who died in hospital were excluded

*Treatment-relevant diseases according to HAS-BLED Score or $\mathrm{CHA}_{2} \mathrm{DS}_{2}$-VASCc-Score

${ }^{a}$ HAS-BLED Score

${ }^{\mathrm{b}} \mathrm{CHA}_{2} \mathrm{DS}_{2}$-VASCc-Score

for several reasons. Treatment pathways developed for the best care of a single disease may interfere with an algorithm developed for another, co-existing disease (disease-disease interaction) [26]. In a Swiss cross-sectional study with patients admitted to an emergency outpatient department, every second patient showed therapeutic conflicts between 
Table 2 Inpatients admitted for the treatment of acute gastrointestinal bleeding (GIB) according to upper and lower GIB

\begin{tabular}{|c|c|c|}
\hline Source of the event according to the ICD-10-code & Description & $n(\%)$ \\
\hline \multicolumn{3}{|l|}{ Upper gastrointestinal bleeding } \\
\hline K92.1 & Melena & $174(15.9)$ \\
\hline K92.2 & Gastrointestinal hemorrhage & $160(14.5)$ \\
\hline K26.0, K26.2 & Acute duodenal ulcer with hemorrhage, w/o perforation & $89(8.1)$ \\
\hline K26.4, K26.6 & Chronic or unspecified duodenal ulcer with hemorrhage, w/o perforation & $83(7.5)$ \\
\hline $\mathrm{K} 25.0, \mathrm{~K} 25.2$ & Acute gastric ulcer with hemorrhage (Ulcus ventriculi), w/o perforation & $68(6.2)$ \\
\hline $\mathrm{K} 25.4, \mathrm{~K} 25.6$ & Chronic or unspecified gastric ulcer with hemorrhage, w/o perforation & $67(6.1)$ \\
\hline K92.0 & Hematemesis & $57(5.2)$ \\
\hline K22.6 & $\begin{array}{l}\text { Gastro-esophageal laceration-hemorrhage syndrome (i.e., Mallory-Weiss } \\
\text { syndrome) }\end{array}$ & $49(4.5)$ \\
\hline K22.8 & Disorders of esophagus (e.g., esophageal bleeding) & $46(4.2)$ \\
\hline K31.82 & $\begin{array}{l}\text { Angiodysplasia of the stomach and duodenum with bleeding (e.g., Dieulafoy's } \\
\text { lesion) }\end{array}$ & $44(4.0)$ \\
\hline K29.0 & Acute hemorrhagic gastritis & $28(2.5)$ \\
\hline K22.3 & Perforation of esophagus & $26(2.4)$ \\
\hline I98.3, I85.0 & Esophageal varices with bleeding & $23(2.1)$ \\
\hline K27.4, K27.6, K28.4, K28.6 & $\begin{array}{l}\text { Chronic or unspecified peptic or gastrojejunal ulcer with hemorrhage, w/o } \\
\text { perforation }\end{array}$ & $12(1.1)$ \\
\hline $\mathrm{K} 27.0, \mathrm{~K} 27.2, \mathrm{~K} 28.0, \mathrm{~K} 28.4$ & Acute peptic or gastrojejunal ulcer with hemorrhage, w/o perforation & $9(0.8)$ \\
\hline \multicolumn{3}{|l|}{ Lower gastrointestinal bleeding } \\
\hline K57.01, K57.11, K57.31, K57.41, K57.51, K57.91 & Diverticulosis of intestine, w/o perforation or abscess with bleeding & $140(12.7)$ \\
\hline K64.0, K64.1, K64.2, K64.3, K64.9 & Hemorrhoids with bleeding & $77(7.0)$ \\
\hline K55.22, K55.82 & Angiodysplasia of the colon (including small intestine): with Bleeding & $62(5.6)$ \\
\hline $\begin{array}{l}\text { K57.03, K57.13, K57.21, K57.23, K57.33, K57.43, } \\
\text { K57.53, K57.93 }\end{array}$ & Diverticulitis of intestine, w/o perforation or abscess with bleeding & $34(3.1)$ \\
\hline K62.5 & Hemorrhage of anus and rectum & $16(1.5)$ \\
\hline
\end{tabular}

the disease and co-existing medication (drug-disease interaction) [27]. One in three of these interactions was serious, possibly leading to life-threatening therapeutic conflicts. Therapeutic conflicts are common among multimorbid patients and merit further research. Management has, however, become more challenging regarding antithrombotic therapy for patients with GIB [28]. The use of antithrombotics in daily clinical practice is indicated for the reduction of the thromboembolic risk, while increasing the risk of bleeding. Antiplatelet medication should be paused depending on the severity of bleeding [29]. However, after endoscopic control of bleeding, medication should be reinstated as soon as possible. The timing should consider the individual cardiovascular risk. Therefore, interdisciplinary work seems to be of particular importance for the management of multimorbid patients with GIB.

In our LGIB patients, atrial fibrillation was associated with an increased risk of 30-day readmissions. As expected, atrial fibrillation is a critical comorbidity requiring a balance between stroke and bleeding risk in decisions on thrombosis prophylaxis [30]. As shown in the ARISTOTLE trial, a history of GIB (especially recent GIB) was associated with increased risk of subsequent, severe GIB in anticoagulated patients with atrial fibrillation [31]. A study conducted in the USA on hospitalized adult patients with atrial fibrillation conducted a cost analysis in relation to occurrence of hospital-associated bleeding events [32]. Results showed that hospital-associated bleedings $(n=2991)$ was attributed to an estimated 8106 additional hospital days and \$16.4 million costs compared with non-bleeders. As indicated by this cost analysis in atrial fibrillation, the economic burden is closely associated with the management of such multimorbid patients. In addition to functional limitations, reduced quality of life and increasing mortality, it has been shown that multimorbidity is associated with high utilization of health services and the resulting costs [33]. As another study demonstrated, an increasing number of risk factors was associated with longer LOS and higher costs [34]. In comparison with other studies, length of stay for GIB was similar to our study [35].

In a prospective study of cancer patients, researchers found an inverse relationship with clinically relevant bleeding during and after hospital stay, therefore recommending further context-specific research [36]. The authors pointed out a therapeutic challenge in the case of cancer patients. After discharge, the risk of bleeding in absence 
Table 3 Upper GI bleeding $(n=791)$

\begin{tabular}{|c|c|c|c|c|}
\hline \multirow[t]{2}{*}{ Variable } & \multicolumn{2}{|c|}{ Length of stay } & \multicolumn{2}{|c|}{$\begin{array}{l}\text { 30-day readmis- } \\
\text { sion }\end{array}$} \\
\hline & $\operatorname{Exp}(B)$ & $(95 \% \mathrm{CI})$ & OR & $(95 \% \mathrm{CI})$ \\
\hline Age, per additional year & 1.00 & $(1.00,1.00)$ & 0.98 & $(0.96,0.99)$ \\
\hline Male sex & 0.96 & $(0.88,1.06)$ & 1.03 & $(0.67,1.56)$ \\
\hline $\begin{array}{l}\text { Number of diagnoses, } \\
\text { per additional diagnosis }\end{array}$ & 1.10 & $(1.08,1.11)$ & 1.05 & $(1.00,1.10)$ \\
\hline Year discharged & 0.97 & $(0.95,0.99)$ & 1.11 & $(1.02,1.20)$ \\
\hline Length of stay & - & - & 1.00 & $(0.97,1.02)$ \\
\hline Alcohol use disorders & 0.91 & $(0.83,1.00)$ & 1.15 & $(0.76,1.74)$ \\
\hline Anemia & 0.94 & $(0.82,1.07)$ & 1.68 & $(1.00,2.84)$ \\
\hline $\begin{array}{l}\text { Atrial fibrillation or } \\
\text { flutter }\end{array}$ & 1.06 & $(0.92,1.23)$ & 1.24 & $(0.67,2.28)$ \\
\hline Cancer & 0.87 & $(0.76,0.995)$ & 2.86 & $(1.68,4.88)$ \\
\hline Chronic kidney disease & 0.80 & $(0.71,0.91)$ & 1.52 & $(0.90,2.56)$ \\
\hline Coagulopathy & 0.96 & $(0.85,1.08)$ & 0.95 & $(0.57,1.58)$ \\
\hline COPD & 1.14 & $(0.96,1.35)$ & 1.57 & $(0.79,3.11)$ \\
\hline Depression & 1.12 & $(0.90,1.40)$ & 1.06 & $(0.42,2.68)$ \\
\hline Diabetes & 0.85 & $(0.76,0.96)$ & 1.41 & $(0.85,2.32)$ \\
\hline Heart failure & 0.91 & $(0.75,1.12)$ & 0.61 & $(0.25,1.45)$ \\
\hline Heart valve disease & 0.93 & $(0.78,1.12)$ & 1.01 & $(0.48,2.15)$ \\
\hline Hypertension & 0.90 & $(0.82,0.996)$ & 1.37 & $(0.89,2.10)$ \\
\hline Liver disease & 1.09 & $(0.94,1.28)$ & 0.92 & $(0.48,1.77)$ \\
\hline Thromboembolic event & 1.27 & $(1.00,1.61)$ & 1.12 & $(0.40,3.12)$ \\
\hline Vascular disease & 0.80 & $(0.72,0.90)$ & 0.94 & $(0.56,1.56)$ \\
\hline Antithrombotic agents & 1.46 & $(1.32,1.62)$ & 1.02 & $(0.65,1.63)$ \\
\hline
\end{tabular}

Numbers in bold indicate statistical significance

Adjusted multivariable linear regression model for the outcome length of stay and adjusted multivariable logistic regression model for the outcome 30-day readmission

95\% CI: 95\% confidence interval; COPD: Chronic obstructive pulmonary disease

of thromboprophylaxis was increased and significantly outweighed that of venous thromboembolism during the hospital stay. Two-thirds of bleeding events were gastrointestinal and were related to gastrointestinal or genitourinary cancer. In our study, cancer was independently associated with 30-day readmissions in both studied populations, UGIB and LGIB. This could partly reflect the therapeutic dilemma of re-bleeding rates. However, to comprehensively interpret the data, it should be noted that we used the ICD-10 classification based on Quan and colleagues to define cancer [21]. This coding algorithm includes any code to identify solid malignant neoplasm, including also tumors of the gastrointestinal tract, but not exclusively.

An interesting observation among UGIB patients was that those with alcohol use disorder showed a trend toward shorter LOS. As other studies found, patients with alcohol use disorder tend to leave the hospitals prematurely against medical advice [37]. Another issue is that alcohol use
Table 4 Lower GI bleeding $(n=310)$

\begin{tabular}{|c|c|c|c|c|}
\hline \multirow[t]{2}{*}{ Variable } & \multicolumn{2}{|c|}{ Length of stay } & \multicolumn{2}{|c|}{$\begin{array}{l}\text { 30-day readmis- } \\
\text { sion }\end{array}$} \\
\hline & $\operatorname{Exp}(B)$ & $(95 \% \mathrm{CI})$ & OR & $(95 \% \mathrm{CI})$ \\
\hline Age, per additional year & 1.01 & $(1.003,1.01)$ & 0.99 & $(0.95,1.02)$ \\
\hline Male sex & 0.95 & $(0.83,1.10)$ & 0.44 & $(0.20,0.99)$ \\
\hline $\begin{array}{l}\text { Number of diagnoses, } \\
\text { per additional diag- } \\
\text { nosis }\end{array}$ & 1.08 & $(1.06,1.10)$ & 1.10 & $(0.99,1.22)$ \\
\hline Year discharged & 0.95 & $(0.93,0.98)$ & 1.05 & $(0.88,1.24)$ \\
\hline Length of stay & - & - & 1.02 & $(0.97,1.07)$ \\
\hline Alcohol use disorders & 1.02 & $(0.79,1.31)$ & 2.43 & $(0.64,9.27)$ \\
\hline Anemia & 0.96 & $(0.79,1.16)$ & 1.98 & $(0.77,5.10)$ \\
\hline $\begin{array}{l}\text { Atrial fibrillation or } \\
\text { flutter }\end{array}$ & 0.95 & $(0.79,1.14)$ & 2.69 & $(1.06,6.82)$ \\
\hline Cancer & 0.98 & $(0.74,1.30)$ & 4.76 & $(1.40,16.20)$ \\
\hline Chronic kidney disease & 1.00 & $(0.82,1.22)$ & 1.15 & $(0.46,2.91)$ \\
\hline Coagulopathy & 0.90 & $(0.76,1.08)$ & 1.21 & $(0.50,2.93)$ \\
\hline COPD & 0.88 & $(0.69,1.12)$ & 0.89 & $(0.25,3.25)$ \\
\hline Depression & 1.03 & $(0.72,1.49)$ & 0.52 & $(0.04,6.35)$ \\
\hline Diabetes & 0.87 & $(0.73,1.04)$ & 0.82 & $(0.31,2.17)$ \\
\hline Heart failure & 1.13 & $(0.84,1.51)$ & 1.01 & $(0.27,3.75)$ \\
\hline Heart valve disease & 0.79 & $(0.63,0.995)$ & 0.53 & $(0.15,1.79)$ \\
\hline Hypertension & 0.95 & $(0.82,1.11)$ & 0.53 & $(0.22,1.25)$ \\
\hline Liver disease & 0.71 & $(0.50,1.01)$ & 0.25 & $(0.02,3.15)$ \\
\hline Thromboembolic event & 0.95 & $(0.57,1.57)$ & 1.14 & $(0.13,9.94)$ \\
\hline Vascular disease & 0.79 & $(0.67,0.94)$ & 1.62 & $(0.62,4.24)$ \\
\hline Antithrombotic agents & 1.25 & $(1.07,1.46)$ & 1.42 & $(0.50,4.03)$ \\
\hline
\end{tabular}

Numbers in bold indicate statistical significance

Adjusted multivariable linear regression model for the outcome length of stay and adjusted multivariable logistic regression model for the outcome 30-day readmission

95\% CI: 95\% confidence interval. COPD: Chronic obstructive pulmonary disease

disorder is a severe and complex comorbidity known for unplanned 30-day readmissions and mortality [38]. Many patients with alcohol use disorder have chronic liver disease and, to some degree, coagulopathy (e.g., prolonged prothrombin time) [39]. It is reasonable to assume in the context of patients with liver disease and alcohol use disorders, that, e.g., the risk of variceal bleeding was increased.

Gastrointestinal bleeding can be a symptom of both, chronic and acute disease, in this patient collective. It would be interesting to investigate how disease clusters change in acute compared with chronic diagnoses and whether this has an impact on LOS and 30-day readmissions. In further analyses, the drug-drug or drug-disease interactions could be considered. Exacerbation of chronic renal failure was common in hospitalized older patients with several pre-existing chronic diseases, while hypertension and atrial fibrillation significantly clustered with adverse drug events [40]. 
Chronic kidney disease was associated with a shorter LOS in patients with UGIB in our study. It would be compelling to study such outcome analyses with a cluster from chronic to acute and acute to chronic diseases. In general, kidney disease is a known risk factor for both, UGIB and LGIB [41]. However, multimorbidity in the narrower sense is not simply the combination of several diseases. The presence of multimorbidity results in independent and complex personal clinical pictures that differ in terms of the characteristics of the individual components [42]. It appears, though, that there are similarities in the clinical picture of GIB. For example, more men than women are affected by GIB [25], as confirmed in the present study.

\section{Limitations}

The findings should be interpreted in the light of the following limitations. This was a single center, retrospective study not designed to evaluate causal relationships-we therefore report independent associations. The patients were not prospectively enrolled for this study and the analyzed EHR data were primarily collected for patient management purposes and not for research. However, only the physicians in charge of the patients were entering the diagnoses into the EHR, only over the course of the patients' stays. After the patients were discharged from the hospital, professional coders assigned ICD-10 codes to each diagnosis, but they neither changed the diagnoses nor their order. A systematic review on the accuracy of routinely collected data and discharge coding concluded that "[...] routinely collected data are sufficiently robust to support their use for research [...]" [43]. Further, we did not use scores to capture the severity of bleeding, i.e., the need for intervention could not be categorized. Finally, no data on deaths after discharge were available, which might have contributed to an underestimation of the 30-day readmission risk. A major strength of this study is the comprehensive and detailed review of the ICD codes that defined gastrointestinal bleeding. There is available evidence that ICD-10 codes applied to an administrative dataset of hospitalized patients appropriately excluded patients without a clinically evident bleeding event [44]. Nevertheless, in certain cases, a chart review is necessary, as conducted in the present study for the control of diagnoses.

An increasingly recognized risk factor for worse clinical outcomes in various patient populations is sarcopenia. In the second half of 2016, nearly seven years after the start of our study period, Anker et al. published the highly cited editorial "Welcome to the ICD-10 code for sarcopenia," [45] additionally supporting the use of routinely collected data for epidemiologic research in the context of sarcopenia. In fact, a recent study by Lattanzi et al. demonstrated that "[...] the rate of refractory variceal bleeding was higher in sarcopenic patients, [...]" [46] a finding that further encourages the future consideration of sarcopenia as an important risk factor also among inpatients, such as those hospitalized for the treatment of GIB.

\section{Conclusion}

Thromboembolic events in UGIB and atrial fibrillation/flutter in LGIB appear to represent clusters of therapeutic conflicts that may negatively impact outcomes in multimorbid patients admitted for the treatment of acute GIB. Further studies are needed to prospectively investigate the impact of these comorbidities, and, importantly, to establish appropriate care and guidelines, especially in patient clusters entailing complex therapeutic conflicts.

Supplementary Information The online version contains supplementary material available at https://doi.org/10.1007/s10620-021-07197-7.

Author's contribution Concept and design: KS, EB, PEB, with contributions by AN. Statistical analyses: KS, with contributions by PEB. Writing of the manuscript: KS, PEB, with revisions by MC. All authors (KS, JB, AN, MC, BUM, EB, PEB) interpreted data, reviewed and critically commented on the manuscript draft and approved the final submitted version of the manuscript.

Funding Open Access funding provided by Universität Zürich. This research received no specific grant from any funding agency in the public, commercial or not-for-profit sectors.

\section{Declarations}

Conflict of interest The authors declare that they have no conflict of interests.

Ethics approval This study had been approved by the Cantonal Ethics Committee Zurich (BASEC 2016-00525), which is the Institutional Review Board (IRB) of the University Hospital Zurich.

Open Access This article is licensed under a Creative Commons Attribution-NonCommercial 4.0 International License, which permits any non-commercial use, sharing, adaptation, distribution and reproduction in any medium or format, as long as you give appropriate credit to the original author(s) and the source, provide a link to the Creative Commons licence, and indicate if changes were made. The images or other third party material in this article are included in the article's Creative Commons licence, unless indicated otherwise in a credit line to the material. If material is not included in the article's Creative Commons licence and your intended use is not permitted by statutory regulation or exceeds the permitted use, you will need to obtain permission directly from the copyright holder. To view a copy of this licence, visit http://creativecommons.org/licenses/by-nc/4.0/. 


\section{References}

1. Garin N, Koyanagi A, Chatterji S, Tyrovolas S, Olaya B, Leonardi $\mathrm{M}$ et al. Global multimorbidity patterns: a cross-sectional, population-based, multi-country study. J Gerontol A Biol Sci Med Sci. 2016;71:205-214.

2. Marengoni A, Roso-Llorach A, Vetrano DL, Fernandez-Bertolin S, Guisado-Clavero M, Violan C et al. Patterns of multimorbidity in a population-based cohort of older people: sociodemographic, lifestyle, clinical, and functional differences. J Gerontol A Biol Sci Med Sci. 2020;75:798-805.

3. van den Akker M, Buntinx F, Metsemakers JF, Roos S, Knottnerus JA. Multimorbidity in general practice: prevalence, incidence, and determinants of co-occurring chronic and recurrent diseases. $J$ Clin Epidemiol. 1998;51:367-375.

4. Aubert CE, Schnipper JL, Roumet M, Marques-Vidal P, Stirnemann J, Auerbach AD et al. Best definitions of multimorbidity to identify patients with high health care resource utilization. Mayo Clin Proc Innov Qual Outcomes. 2020;4:40-49.

5. Battegay E. Multimorbidity is a game changer. Swiss Med Wkly. 2019;149:w20131.

6. Poblador-Plou B, Calderon-Larranaga A, Marta-Moreno J, Hancco-Saavedra J, Sicras-Mainar A, Soljak M et al. Comorbidity of dementia: a cross-sectional study of primary care older patients. BMC Psychiatry. 2014;14:84.

7. World Health Organization W. Life expectancy and Healthy life expectancy Data by WHO region. Downloaded at: https://apps. who.int/gho/data/view.main.SDG2016LEXREGv?lang=en. Accessed on 08-04-2020.

8. Fortin M, Stewart M, Poitras ME, Almirall J, Maddocks H. A systematic review of prevalence studies on multimorbidity: toward a more uniform methodology. Ann Fam Med. 2012;10:142-151.

9. Excoffier S, Herzig L, N'Goran AA, Deruaz-Luyet A, Haller DM. Prevalence of multimorbidity in general practice: a cross-sectional study within the Swiss Sentinel Surveillance System (Sentinella). BMJ Open. 2018;8:e019616.

10. Kowal P, Arokiasamy P, Afshar S, Pati S, Snodgrass JJ. Multimorbidity: health care that counts "past one" for 1.2 billion older adults. Lancet. 2015;385:2252-2253.

11. Tanne JH, Hayasaki E, Zastrow M, Pulla P, Smith P, Rada AG. Covid-19: how doctors and healthcare systems are tackling coronavirus worldwide. BMJ. 2020;368:m1090.

12. Beeler PE, Cheetham M, Held U, Battegay E. Depression is independently associated with increased length of stay and readmissions in multimorbid inpatients. Eur J Intern Med. 2020;73:59-66.

13. Wuerth BA, Rockey DC. Changing epidemiology of upper gastrointestinal hemorrhage in the last decade: a nationwide analysis. Dig Dis Sci. 2018;63:1286-1293. https://doi.org/10.1007/ s10620-017-4882-6.

14. Strate LL. Lower GI bleeding: epidemiology and diagnosis. Gastroenterol Clin North Am. 2005;34:643-664.

15. Stanley AJ, Laine L. Management of acute upper gastrointestinal bleeding. BMJ. 2019;364:1536.

16. Fearnhead N. Acute lower gastrointestinal bleeding. Medicine. 2015;43.

17. von Elm E, Altman DG, Egger M, Pocock SJ, Gotzsche PC, Vandenbroucke JP, et al. The Strengthening the Reporting of Observational Studies in Epidemiology (STROBE) statement: guidelines for reporting observational studies. PLoS Med. 2007;4:e296.

18. Rockall TA, Logan RF, Devlin HB, Northfield TC. Incidence of and mortality from acute upper gastrointestinal haemorrhage in the United Kingdom. Steering Committee and members of the National Audit of Acute Upper Gastrointestinal Haemorrhage. BMJ. 1995;311:222-226.
19. Pisters R, Lane DA, Nieuwlaat R, de Vos CB, Crijns HJ, Lip GY. A novel user-friendly score (HAS-BLED) to assess 1-year risk of major bleeding in patients with atrial fibrillation: the Euro Heart Survey. Chest. 2010;138:1093-1100.

20. Lip GY, Nieuwlaat R, Pisters R, Lane DA, Crijns HJ. Refining clinical risk stratification for predicting stroke and thromboembolism in atrial fibrillation using a novel risk factor-based approach: the euro heart survey on atrial fibrillation. Chest. 2010;137:263-272.

21. Quan H, Sundararajan V, Halfon P, Fong A, Burnand B, Luthi JC et al. Coding algorithms for defining comorbidities in ICD-9-CM and ICD-10 administrative data. Med Care. 2005;43:1130-1139.

22. Barnett K, Mercer SW, Norbury M, Watt G, Wyke S, Guthrie B. Epidemiology of multimorbidity and implications for health care, research, and medical education: a cross-sectional study. Lancet. 2012;380:37-43.

23. Sharabiani MT, Aylin P, Bottle A. Systematic review of comorbidity indices for administrative data. Med Care. 2012;50:1109-1118.

24. van Leerdam ME. Epidemiology of acute upper gastrointestinal bleeding. Best Pract Res Clin Gastroenterol. 2008;22:209-224.

25. Oakland K. Changing epidemiology and etiology of upper and lower gastrointestinal bleeding. Best Pract Res Clin Gastroenterol. 2019;42-43:101610.

26. Battegay E, Cheetham M, Holzer BM, Nowak A, Schmidt D, Rampini S. Multimorbidity management and the physician's daily clinical dilemma. Internist (Berl). 2017;58:344-353.

27. Markun S, Holzer BM, Rodak R, Kaplan V, Wagner CC, Battegay E, et al. Therapeutic conflicts in emergency department patients with multimorbidity: a cross-sectional study. PLoS One. 2014;9:e110309.

28. Schizas D, Kariori M, Boudoulas KD, Siasos G, Patelis N, Kalantzis $\mathrm{C}$ et al. Management of antithrombotic therapy in patients with coronary artery disease or atrial fibrillation who underwent abdominal surgical operations. Curr Pharm Des. 2018;24:2743-2755.

29. Jansen P, Götz M. Trebicka J [S2k-Leitlinie Gastrointestinale Blutung. AWMF-Register Nr. 021-028]. Z Gastroenterol 2017;55:937-946.

30. Apostolakis S, Lane DA, Buller H, Lip GY. Comparison of the CHADS2, CHA2DS2-VASc and HAS-BLED scores for the prediction of clinically relevant bleeding in anticoagulated patients with atrial fibrillation: the AMADEUS trial. Thromb Haemost. 2013;110:1074-1079.

31. Garcia DA, Fisher DA, Mulder H, Wruck L, De Caterina R, Halvorsen $\mathrm{S}$ et al. Gastrointestinal bleeding in patients with atrial fibrillation treated with Apixaban or warfarin: Insights from the Apixaban for Reduction in Stroke and Other Thromboembolic Events in Atrial Fibrillation (ARISTOTLE) trial. Am Heart $J$. 2020;221:1-8.

32. Amin AN, Robinson SB, Bowdy BD, Jing Y, Johnson BH, Wiederkehr DP. Relationship of hospital-associated bleeding with length of stay and total hospitalization costs in patients hospitalized for atrial fibrillation. J Med Econ. 2016;19:490-496.

33. Violan C, Foguet-Boreu Q, Flores-Mateo G, Salisbury C, Blom J, Freitag M, et al. Prevalence, determinants and patterns of multimorbidity in primary care: a systematic review of observational studies. PLoS One. 2014;9:e102149.

34. Saltzman JR, Tabak YP, Hyett BH, Sun X, Travis AC, Johannes RS. A simple risk score accurately predicts in-hospital mortality, length of stay, and cost in acute upper GI bleeding. Gastrointest Endosc. 2011;74:1215-1224.

35. Whelan CT, Chen C, Kaboli P, Siddique J, Prochaska M, Meltzer DO. Upper versus lower gastrointestinal bleeding: a direct comparison of clinical presentation, outcomes, and resource utilization. J Hosp Med. 2010;5:141-147. 
36. Di Nisio M, Candeloro M, Rutjes AWS, Galli V, Tritto M, Porreca E. Bleeding and venous thromboembolic events in patients with active cancer hospitalized for an acute medical illness. Thromb Res. 2018;169:44-49.

37. Saitz R, Ghali WA, Moskowitz MA. The impact of leaving against medical advice on hospital resource utilization. J Gen Intern Med. 2000;15:103-107.

38. Gili-Miner M, Bejar-Prado L, Gili-Ortiz E, Ramirez-Ramirez G, Lopez-Mendez J, Lopez-Millan JM et al. Alcohol use disorders among surgical patients: unplanned 30-days readmissions, length of hospital stay, excessive costs and mortality. Drug Alcohol Depend. 2014;137:55-61.

39. Jutabha R, Jensen DM. Management of upper gastrointestinal bleeding in the patient with chronic liver disease. Med Clin North Am. 1996;80:1035-1068.

40. Buurman BM, Frenkel WJ, Abu-Hanna A, Parlevliet JL, de Rooij SE. Acute and chronic diseases as part of multimorbidity in acutely hospitalized older patients. Eur J Intern Med. 2016;27:68-75.

41. Wasse H, Gillen DL, Ball AM, Kestenbaum BR, Seliger SL, Sherrard D et al. Risk factors for upper gastrointestinal bleeding among end-stage renal disease patients. Kidney Int. 2003;64:1455-1461.

42. Bulletin der SAMW SAdMW. [Multimorbidität: Eine Herausforderung der Neuzeit.] Available at: https://www.samw.ch/dam/ jcr:9195033a-a6bf-4655-8414-961b97a40028/bulletin_samw_ 14_4.pdf. SAMW Bulletin 2014;04.

43. Burns EM, Rigby E, Mamidanna R, Bottle A, Aylin P, Ziprin P et al. Systematic review of discharge coding accuracy. $J$ Public Health (Oxf). 2012;34:138-148.

44. Joos C, Lawrence K, Jones AE, Johnson SA, Witt DM. Accuracy of ICD-10 codes for identifying hospitalizations for acute anticoagulation therapy-related bleeding events. Thromb Res. 2019;181:71-76.

45. Anker SD, Morley JE, von Haehling S. Welcome to the ICD10 code for sarcopenia. J Cachexia Sarcopenia Muscle. 2016;7:512-514.

46. Lattanzi B, Gioia S, Di Cola S, D’Ambrosio D, Nardelli S, Tavano $\mathrm{D}$ et al. Prevalence and impact of sarcopenia in non-cirrhotic portal hypertension. Liver Int. 2019;39:1937-1942.

Publisher's Note Springer Nature remains neutral with regard to jurisdictional claims in published maps and institutional affiliations. 